\title{
Front Matter: Volume 6567
}

, "Front Matter: Volume 6567," Proc. SPIE 6567, Signal Processing, Sensor Fusion, and Target Recognition XVI, 656701 (16 May 2007); doi:

10.1117/12.741108

SPIE Event: Defense and Security Symposium, 2007, Orlando, Florida, United States 


\title{
PROCEEDINGS OF SPIE
}

\section{Signal Processing, Sensor Fusion, and Target Recognition XVI}

\author{
Ivan Kadar \\ Editor
}

9-11 April 2007

Orlando, Florida, USA

Sponsored and Published by

SPIE-The International Society for Optical Engineering

Volume 6567

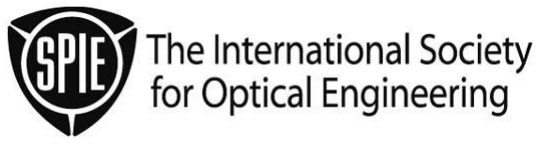

Proceedings of SPIE-The International Society for Optical Engineering, 9780819466891, v. 6567

SPIE is an international technical society dedicated to advancing engineering and scientific applications of optical, photonic, imaging, electronic, and optoelectronic technologies. 
The papers included in this volume were part of the technical conference cited on the cover and title page. Papers were selected and subject to review by the editors and conference program committee. Some conference presentations may not be available for publication. The papers published in these proceedings reflect the work and thoughts of the authors and are published herein as submitted. The publisher is not responsible for the validity of the information or for any outcomes resulting from reliance thereon.

Please use the following format to cite material from this book:

Author(s), "Title of Paper," in Signal Processing, Sensor Fusion, and Target Recognition XVI, edited by Ivan Kadar, Proceedings of SPIE Vol. 6567 (SPIE, Bellingham, WA, 2007) Article CID Number.

ISSN 0277-786X

ISBN 9780819466891

Published by

SPIE-The International Society for Optical Engineering

P.O. Box 10, Bellingham, Washington 98227-0010 USA

Telephone 1 360/676-3290 (Pacific Time) · Fax 1 360/647-1445

http://www.spie.org

Copyright @ 2007 , The Society of Photo-Optical Instrumentation Engineers

Copying of material in this book for internal or personal use, or for the internal or personal use of specific clients, beyond the fair use provisions granted by the U.S. Copyright Law is authorized by SPIE subject to payment of copying fees. The Transactional Reporting Service base fee for this volume is $\$ 18.00$ per article (or portion thereof), which should be paid directly to the Copyright Clearance Center (CCC), 222 Rosewood Drive, Danvers, MA 01923. Payment may also be made electronically through CCC Online at http://www.copyright.com. Other copying for republication, resale, advertising or promotion, or any form of systematic or multiple reproduction of any material in this book is prohibited except with permission in writing from the publisher. The CCC fee code is 0277 $786 \times / 07 / \$ 18.00$.

Printed in the United States of America. 


\section{Contents}

ix Conference Committee

\section{MULTISENSOR FUSION, MULTITARGET TRACKING, AND RESOURCE MANAGEMENT I}

656702 Impact and point prediction using a neural extended Kalman filter with multiple sensors [6567-01]

S. C. Stubberud, Rockwell-Collins (USA); K. A. Kramer, Univ. of San Diego (USA)

656703 Markov chains for the prediction of tracking performance [6567-02]

P. O. Arambel, M. Antone, BAE Systems, Advanced Information Technologies (USA)

656704 Two solutions to the localization using time difference of arrival problem [6567-03]

T. Sathyan, T. Kirubarajan, McMaster Univ. (Canada)

656705 Acoustic signature analysis and data fusion of vehicles based on acoustic sensor arrays [6567-04]

T. Viangteeravat, A. Shirkhodaie, H. Rababaah, Tennessee State Univ. (USA)

656706 Structural and metric correlation of electro-optical and radar-generated tracks [6567-05]

B. Kovalerchuk, Central Washington Univ. (USA)

656707 Fast video target tracking in the presence of occlusion and camera motion blur [6567-06]

C. Li, B. Li, J. Si, Arizona State Univ. (USA); G. P. Abousleman, General Dynamics C4 Systems (USA)

MULTISENSOR FUSION, MULTITARGET TRACKING, AND RESOURCE MANAGEMENT II

656708 Fuzzy decision trees for planning and autonomous control of a coordinated team of UAVs [6567-07]

J. F. Smith III, T. H. Nguyen, Naval Research Lab. (USA)

Pagination: Proceedings of SPIE follow an e-First publication model, with papers published first online and then in print and on CD-ROM. Papers are published as they are submitted and meet publication criteria. A unique, consistent, permanent citation identifier (CID) number is assigned to each article at the time of the first publication. Utilization of CIDs allows articles to be fully citable as soon they are published online, and connects the same identifier to all online, print, and electronic versions of the publication.

SPIE uses a six-digit CID article numbering system in which:

- The first four digits correspond to the SPIE volume number.

- The last two digits indicate publication order within the volume using a Base 36 numbering system employing both numerals and letters. These two-number sets start with $00,01,02,03,04,05,06,07,08,09,0 A, 0 B \ldots$. OZ, followed by 10-1Z, 20-2Z, etc.

The CID number appears on each page of the manuscript. The complete citation is used on the first page, and an abbreviated version on subsequent pages. 
656709 Random set tracking and entropy-based control applied to distributed sensor networks [6567-08]

D. Stein, J. Witkoskie, S. Theophanis, W. Kuklinski, The MITRE Corp. (USA)

65670A Adaptive sensor tasking using genetic algorithms [6567-09]

P. J. Shea, J. Kirk, D. Welchons, Black River Systems Co. (USA)

65670B Distributed sensor resource management and planning [6567-10]

D. Khosla, J. Guillochon, HRL Labs. LLC (USA)

65670C Integrated tracking and sensor management based on expected information gain [6567-11]

K. A. B. White, Defence Science and Technology Organisation (Australia)

65670D Distributed simulation of an information-based sensor management system [6567-12]

J. Malachowski, George Mason Univ. (USA)

\section{ASSISTED TARGET RECOGNITION (ATR) I}

65670E UAV-based distributed ATR under realistic simulated environmental effects [6567-13]

X. Chen, S. Gong, N. A. Schmid, M. C. Valenti, West Virginia Univ. (USA)

65670F Real-time vehicle detection in infrared and visible images [6567-14]

C. YU, A. Dong, Z. Yue, P. Topiwala, FastVDO LLC (USA)

$65670 \mathrm{I}$ Information theoretic partitioning and confidence based weight assignment for multiclassifier decision level fusion in hyperspectral target recognition applications [6567-15] S. Prasad, L. M. Bruce, Mississippi State Univ. (USA)

65670H Applying target shadow models for SAR ATR [6567-16]

S. Papson, R. M. Narayanan, The Pennsylvania State Univ. (USA)

\section{ASSISTED TARGET RECOGNITION (ATR) II}

65670 Detection and identification of human targets in radar data [6567-17]

S. Z. Gürbüz, Georgia Institute of Technology (USA); W. L. Melvin, Georgia Tech Research Institute (USA); D. B. Williams, Georgia Institute of Technology (USA)

65670 J Improving throughput for temporal target nomination using existing infrastructure [6567-18] P. G. Raeth, Ball Aerospace \& Technologies Corp. (USA)

65670K Time-frequency transform techniques for seabed and buried target classification [6567-19] M. Barbu, E. Kaminsky, R. E. Trahan, Jr., Univ. of New Orleans (USA)

$65670 \mathrm{M}$ Face and automatic target recognition based on super-resolution class-specific subspace [6567-63]

W. Asdornwised, Chulalongkorn Univ. (Thailand) 
65670N Invited panel discussion: Issues and challenges in uncertainty representation and management with applications to real-world problems (Invited Paper) [6567-65] I. Kadar, Interlink Systems Sciences, Inc. (USA); E. H. Ruspini, Artificial Intelligence Ctr., SRI International (USA); A.-L. Jousselme, P. Maupin, R\&D Defence Canada/Valcartier (Canada); R. Mahler, Lockheed Martin MS2 Tactical Systems (USA); J. S. J. Peri, Johns Hopkins Univ. Applied Physics Lab. (USA); P. Valin, Defence R\&D Canada/Valcartier (Canada); D. Akselrod, A. Sinha, T. Kirubarajan, McMaster Univ. (Canada)

\section{MULTISENSOR FUSION METHODOLOGIES AND APPLICATIONS I}

656700 A survey of PHD filter and CPHD filter implementations [6567-21]

R. Maher, Lockheed Martin MS2 Tactical Systems (USA)

65670P A unified Bayesian theory of measurements [6567-22]

R. Maher, Lockheed Martin MS2 Tactical Systems (USA)

65670Q Mission-based situational awareness sensor management and information fusion [6567-23] A. El-Fallah, A. Zatezalo, Scientific Systems Co., Inc. (USA); R. Mahler, Lockheed Martin Tactical Defense Systems (USA); M. Alford, Air Force Research Lab. (USA)

65670R Space-based sensor management and geostationary satellites tracking [6567-24] A. El-Fallah, A. Zatezalo, Scientific Systems Co., Inc. (USA); R. Mahler, Lockheed Martin Tactical Defense Systems (USA); R. K. Mehra, Scientific Systems Co., Inc. (USA); D. Donatelli, Air Force Research Lab. (USA)

$65670 S$ PFLib: an object-oriented MATLAB toolbox for particle filtering [6567-25]

L. Chen, Scientific Systems Co., Inc. (USA); C. Lee, A. Budhiraja, Univ. of North Carolina at Chapel Hill (USA); R. K. Mehra, Scientific Systems Co., Inc. (USA)

\section{MULTISENSOR FUSION METHODOLOGIES AND APPLICATIONS II}

$65670 \mathrm{~A}$ A testbed for architecture and fidelity trade studies in the Bayesian decision-level fusion of ATR products [6567-27]

K. J. Erickson, Jacobs Technology (USA); T. D. Ross, Air Force Research Lab. (USA)

$65670 \mathrm{~V}$ Survey of approaches and experiments in decision-level fusion of automatic target recognition (ATR) products [6567-28]

T. D. Ross, Air Force Research Lab. (USA); D. R. Morgan, BAE consultant (USA); E. P. Blasch, Air Force Research Lab. (USA); K. J. Erickson, Jacobs Engineering (USA); B. D. Kahler, General Dynamics (USA)

65670W ROC curve formulas for fused correlated classification systems [6567-29]

M. E. Oxley, S. N. Thorsen, Air Force Institute of Technology (USA); C. M. Schubert, Virginia Commonwealth Univ. (USA) 
65670 An improved DS acoustic-seismic modality fusion algorithm based on a new cascaded fuzzy classifier for ground-moving targets classification in wireless sensor networks [6567-58]

Q. Pan, J. Wei, H. Cao, N. Li, H. Liu, Shanghai Institute of Microsystem and Information Technology (China)

\section{MULTISENSOR FUSION METHODOLOGIES AND APPLICATIONS III}

$65670 Z$ Aerial video and ladar imagery fusion for persistent urban vehicle tracking [6567-30]

P. Cho, D. Greisokh, H. Anderson, J. Sandland, R. Knowlton, MIT Lincoln Lab. (USA)

656710 Advanced activity reporting in a multi-layered unattended ground sensor network [6567-31]

T. W. Joslin, Harris Corp. (USA)

656711 The use of optical RF network for target detection and tracking [6567-32]

M.-C. Li, Liceimer (USA)

\section{MULTISENSOR FUSION METHODOLOGIES AND APPLICATIONS IV}

656712 Issues and challenges in resource management and its interaction with levels $2 / 3$ fusion with applications to real-world problems: an annotated perspective [6567-33]

E. Blasch, Air Force Research Lab. (USA); I. Kadar, Interlink Systems Sciences, Inc. (USA); K. Hintz, George Mason Univ. (USA); J. Biermann, FGAN-FKIE (Germany); C.-Y. Chong, BAE Systems (USA); J. Salerno, Air Force Research Lab. (USA); S. Das, Charles River Analytics (USA)

656713 Game theoretic behavior features change prediction in hostile environments [6567-34] M. Wei, Intelligent Automation Inc. (USA); E. Blasch, Air Force Research Lab. (USA); G. Chen, Intelligent Automation Inc. (USA); J. B. Cruz, Jr., The Ohio State Univ. (USA); L. Haynes, Intelligent Automation, Inc. (USA); M. Kruger, Office of Naval Research (USA); I. Sityar, Alion Science and Technology (USA)

656714 Statistical comparison of a hybrid approach with approximate and exact inference models for Fusion 2+ [6567-35]

K. D. Lee, E. Wiesenfeld, A. Gelfand, Decisive Analytics Corp. (USA)

656715 Hybrid methodology for situation assessment model development within an air operations center domain [6567-36]

S. Ho, P. Gonsalves, C. Call, Charles River Analytics (USA)

\section{SIGNAL AND IMAGE PROCESSING I}

656716 Automatic building detection and 3D shape recovery from single monocular electro-optic imagery [6567-38]

D. A. Lavigne, Defence R\&D Canada/Valcartier (Canada); P. Saeedi, Simon Fraser Univ. (Canada); A. Dlugan, N. Goldstein, H. Zwick, MacDonald, Dettwiler \& Associates (Canada) 
656717 Application of a dynamic feature selection algorithm to multi-sensor image registration [6567-39]

S. DelMarco, V. Tom, H. Webb, D. Lefebvre, BAE Systems Inc. (USA)

656718 A verification metric for multi-sensor image registration [6567-40]

S. DelMarco, V. Tom, H. Webb, D. Lefebvre, BAE Systems Inc. (USA)

656719 Detecting change in images with parallax [6567-41]

M. J. Carlotto, General Dynamics (USA)

65671A Feature-based anomaly detection [6567-42]

M. J. Carlotto, General Dynamics Advanced Information Systems (USA)

65671B Target detection based on multiresolution fractal analysis [6567-43]

D. Charalampidis, G. W. Stein, Univ. of New Orleans (USA)

65671E A spectral independent morphological adaptive classifier [6567-46]

J. B. Montgomery, C. T. Montgomery, M\&M Aviation (USA); R. B. Sanderson,

J. F. McCalmont, Air Force Research Lab. (USA)

65671F Adaptive target segmentation using runtime-weighted features [6567-60]

J.-H. Jung, H.-S. Lee, D.-J. Park, Korea Advanced Institute of Science and Technology

(South Korea); C.-H. Park, J.-I. Lee, Samsung Thales Co., Ltd. (South Korea)

$65671 \mathrm{G}$ Real-time color transfer system for low-light level visible and infrared images in YUV color space [6567-61]

L. Wang, Y. Zhao, W. Jin, S. Shi, S. Wang, Beijing Institute of Technology (China)

SIGNAL AND IMAGE PROCESSING II

65671H A fuzzy rule base system for object-based feature extraction and classification [6567-47]

X. Jin, S. Paswaters, ITT Visual Information Solutions (USA)

656711 Novel lock-in amplifier for identification of luminescent materials for authentication [6567-48]

A. D. McAulay, Lehigh Univ. (USA)

$65671 \mathrm{~J}$ Robust modulation classification techniques using cumulants and hierarchical neural networks [6567-49]

J. A. DeClovet, M. Naraghi-Pour, Louisiana State Univ. (USA)

65671K Real-time 3D target tracking and localization for arbitrary camera geometries [6567-50] A. P. Kulkarni, Arizona State Univ. (USA); G. P. Abousleman, General Dynamics Decision Systems (USA); J. Si, Arizona State Univ. (USA)

65671L Development and results of NIR polarization camera [6567-51]

G. Baker, M. Wilson, P. Coulter, MilSys Technologies, LLC (USA)

65671M MTF for Bayer pattern color detector [6567-52]

E. Yotam, P. Ephi, Y. Ami, Rafael Ltd. (Israel) 
$65671 \mathrm{~N}$ Signal processing techniques for heterodyne differential absorption lidar [6567-53] J. Y. Beyon, California State Univ. (USA); G. J. Koch, S. Ismail, NASA Langley Research Ctr. (USA)

656710 Dwi-detector optical sensor for measuring the concentration of total suspended solids in waters [6567-57]

S. G. Daraigan, Univ. Sains Malaysia (Malaysia) and Hadhramout Univ. of Science and Technology (Yemen); M. Z. MatJafri, K. Abdullah, H. S. Lim, S. A. Hashim, Univ. Sains Malaysia (Malaysia)

65671P Effective improvement on traditional filter to reduce envelope delay [6567-59]

Z. Liang, B. Li, J. Wei, Y. Liu, H. Liu, Shanghai Institute of Microsystem and Information Technology (China)

$65671 Q$ Radar detection using sparsely distributed apertures in urban environment [6567-62] I.-Y. Son, T. Varslot, C. E. Yarman, Rensselaer Polytechnic Institute (USA); A. Pezeshki, Princeton Univ. (USA); B. Yazici, M. Cheney, Rensselaer Polytechnic Institute (USA)

Author Index 


\title{
Conference Committee
}

\author{
Symposium Chair
}

John C. Carrano, Luminex Corporation (USA)

Symposium Cochair

Larry B. Stotts, Defense Advanced Research Projects Agency (USA)

Program Track Chair

Ivan Kadar, Interlink Systems Sciences, Inc. (USA)

Conference Chair

Ivan Kadar, Interlink Systems Sciences, Inc. (USA)

Program Committee

Mark G. Alford, Air Force Research Laboratory (USA)

Erik P. Blasch, Air Force Research Laboratory (USA)

Mark J. Carlotto, General Dynamics, Advanced Information Systems (USA)

Kuo Chu Chang, George Mason University (USA)

Chee-Yee Chong, BAE Systems Advanced Information Technologies (USA)

Marvin N. Cohen, Georgia Tech Research Institute (USA)

Mohammad Farooq, Royal Military College of Canada (Canada)

Charles W. Glover, Oak Ridge National Laboratory (USA)

I. R. Goodman, Space and Naval Warfare Systems Center, San Diego (USA)

Lynne L. Grewe, California State University/East Bay (USA)

Michael L. Hinman, Air Force Research Laboratory (USA)

Kenneth J. Hintz, George Mason University (USA)

Jon S. Jones, Air Force Research Laboratory (USA)

Thiagalingam Kirubarajan, McMaster University (Canada)

Martin E. Liggins II, The MITRE Corporation (USA)

Perry C. Lindberg, Teledyne Brown Engineering (USA)

James Llinas, SUNY/University at Buffalo (USA)

Ronald P. Mahler, Lockheed Martin MS2 Tactical Systems (USA)

Raj P. Malhotra, Air Force Research Laboratory (USA)

Alastair D. McAulay, Lehigh University (USA)

Raman K. Mehra, Scientific Systems Company, Inc. (USA)

Harley R. Myler, Lamar University (USA)

David Nicholson, BAE Systems plc (United Kingdom)

Leslie M. Novak, BAE Systems Advanced Information Technologies (USA)

Andrew G. Tescher, AGT Associates (USA) 
Stelios C. Thomopoulos, National Center for Scientific Research (Greece)

Wiley E. Thompson, New Mexico State University (USA)

Session Chairs

1 Multisensor Fusion, Multitarget Tracking, and Resource Management I Ivan Kadar, Interlink Systems Sciences, Inc. (USA)

Kenneth J. Hintz, George Mason University (USA)

2 Multisensor Fusion, Multitarget Tracking, and Resource Management II

Kenneth J. Hintz, George Mason University (USA)

Ivan Kadar, Interlink Systems Sciences, Inc. (USA)

3 Assisted Target Recognition (ATR) I

Ivan Kadar, Interlink Systems Sciences, Inc. (USA)

Kenneth J. Hintz, George Mason University (USA)

$4 \quad$ Assisted Target Recognition (ATR) II

Ivan Kadar, Interlink Systems Sciences, Inc. (USA)

Kenneth J. Hintz, George Mason University (USA)

5 Multisensor Fusion Methodologies and Applications I

Ronald P. Mahler, Lockheed Martin MS2 Tactical Systems (USA)

6 Multisensor Fusion Methodologies and Applications II

Ivan Kadar, Interlink Systems Sciences, Inc. (USA)

Michael L. Hinman, Air Force Research Laboratory (USA)

7 Multisensor Fusion Methodologies and Applications III

Michael L. Hinman, Air Force Research Laboratory (USA)

Ivan Kadar, Interlink Systems Sciences, Inc. (USA)

8 Multisensor Fusion Methodologies and Applications IV

Michael L. Hinman, Air Force Research Laboratory (USA)

Ivan Kadar, Interlink Systems Sciences, Inc. (USA)

$9 \quad$ Signal and Image Processing I

Lynne L. Grewe, California State University/East Bay (USA)

Ivan Kadar, Interlink Systems Sciences, Inc. (USA)

10 Signal and Image Processing II

Alastair D. McAulay, Lehigh University (USA)

Lynne L. Grewe, California State University/East Bay (USA) 